\title{
Bradycardic Effects of AQ-A 39 (Falipamil) in Situ and in Isolated, Blood-Perfused Dog Hearts Comparison with Alinidine and Verapamil
}

\author{
Yasuhiro Ogrwara, M.D., Yasuyuki Furukawa, M.D., \\ Kunio Akahane, M.D., Masayuki Haniuda, M.D., \\ and Shigetoshi CHIBA, M.D.
}

\section{Summary}

The cardiovascular effects of a specific bradycardic agent, AQ-A 39, were investigated in intact donor dogs and isolated and cross-perfused dog heart preparations. Intravenous administration of AQ-A 39 (10$1000 \mu \mathrm{g} / \mathrm{kg}$ ) to the donor $\mathrm{dog}$ caused a dose-dependent heart rate decrease in the donor dog and a decreased atrial rate in the isolated atrium perfused by the donor's blood. The arterial blood pressure of the donor dog and contractile force of the atrial preparation were unchanged or slightly decreased. The direct injection of AQ-A 39 (1-300 $\mu$ g) into the sinus node artery of the isolated atrium caused dose-dependent negative chronotropic and slight, transient positive inotropic responses. Alinidine and verapamil caused marked negative chronotropic and inotropic responses. The negative chronotropic effect of AQ-A 39 was not modified by atropine. However, it was enhanced slightly but significantly by propranolol, indicating that AQ-A 39-induced bradycardia was antagonized partly by beta-adrenoceptor function. These results confirmed that AQ-A 39 selectively reduced sinus rate by a direct action on the sinus node. Furthermore, the potency of the bradycardic action, compared with the decrease in contractility, was greater than for alinidinc or vcrapamil. AQ-A $39(300 \mu \mathrm{g})$ tended to depress norepinephrine (NE)-induced positive chronotropic but not inotropic effects in isolated atria. By contrast, verapamil $(3-10 \mu \mathrm{g})$ significantly depressed the NE-induced positive inotropic but not the chronotropic effect, and propranolol (10 $\mu \mathrm{g})$ suppressed both cardiac effects of NE. These data suggest that the AQ-A 39-induced, selective attenuation of the NE-induced chronotropic effect is not due to either calcium channel blockade or beta-adrenoceptor antagonism.

\section{Additional Indexing Words:}

AQ-A 39 Alinidine Isolated dog atrium Bradycardia Propranolol Verapamil Japan.

From the Department of Pharmacology, Shinshu University School of Medicine, Matsumoto,

Address for reprints: Shigetoshi Chiba, M.D., Department of Pharmacology, Shinshu University School of Medicine, Matsumoto 390, Japan.

Received for publication January 18, 1988.

Accepted April 25, 1988. 
Q-A 39, a derivative of verapamil, is a bradycardic agent that predomiA nantly decreases heart rate in comparison with cardiac contractility and atrioventricular conductivity. ${ }^{1-3)}$ Although AQ-A 39 rcadily induced a negative chronotropic effect, an inotropic effect was inconsistent. Dammgen et $\mathrm{al}^{1 / 1}$ and Kawada et $\mathrm{al}^{{ }^{3 /}}$ demonstrated that AQ-A 39, at higher doses, induced a negative inotropic effect in isolated or anesthetized mammalian hearts. On the other hand, Urthaler and Walker") observed that AQ-A 39 elicited a positive inotropic response which was diminished in part by a beta-adrenoceptor antagonist, propranolol. Sys et $\mathrm{a}^{5 \mathrm{5}}$ also observed that, at lower doses, AQ-A 39 induced a minute positive inotropic action on mammalian ventricular muscles but that at higher doses myocardial contractility decreased. In the open-chest, anesthetized dog, intravenous administration of AQ-A 39 decreased heart rate without affecting left ventricular $\mathrm{dP} / \mathrm{dt}$, while alinidine, another bradycardic agent, decreased both heart rate and left ventricular $\mathrm{dP} / \mathrm{dt}^{6}{ }^{6}$ Although the mechanisms of the bradycardic actions of AQ-A 39 are not completely understood, it was reported that AQ-A 39 inhibited a slow inward current in rabbit heart preparations. ${ }^{7,8}$ ) However, the involvement of beta-adrenergic or muscarinic receptors was excluded for the mechanisms of the AQ-A 39-induced bradycardia in isolated guinea pig atria. ${ }^{2), 91}$

Recently, we studied the bradycardic effects of alinidine injected intravenously to the support $\operatorname{dog}$ or intraarterially to the isolated atria using isolated, cross-circulated dog heart preparations. ${ }^{10)}$ Alinidine induced a greater negative chronotropic effect than a negative inotropic effect in isolated dog atria. Thus, in the present study, we examined the cardiovascular effects of AQ-A 39 administered intravenously in the anesthetized donor dog. The chronotropic and inotropic effects of AQ-A 39, alinidine and verapamil injected intraarterially were then compared in the isolated, perfused atrial preparation.

It was reported that AQ-A 39 attenuated catecholamine-induced positive chronotropic, but not inotropic effects in isolated mammalian hearts.9) However, the mechanism of the AQ-A 39-induced selective attenuation of catecholamine-induced positive chronotropic effect is unclear. Thus, we also investigated the selective attenuation of the catecholamine-induced positive chronotropic effect in the isolated, blood-perfused dog atrial preparations.

\section{Methods}

The isolated right atria were obtained from 24 mongrel dogs weighing 8 to $23 \mathrm{~kg}$. Each dog was anesthetized intravenously with $30 \mathrm{mg} / \mathrm{kg}$ of sodium 
pentobarbital. After administration of 200 USP units $/ \mathrm{kg}$ of sodium heparin, the right atrium was excised and immersed in cold Tyrode's solution. The wet weight of the isolated right atrium varied from 6 to $12 \mathrm{~g}$. Twenty-four support dogs (9 to $27 \mathrm{~kg}$ ) were also anesthetized with sodium pentobarbital and administered sodium heparin (500 USP units $/ \mathrm{kg}$ i.v.) at the beginning of the perfusion. Support dogs were artificially ventilated with room air using a Harvard respirator (model 607). The isolated right atrium was perfused through the cannulated sinus node artery via the right coronary artery with heparinized arterial blood led from the left carotid artery of the support dog with the aid of a peristaltic pump (Harvard Apparatus, model 1201). A pneumatic resistance was placed in parallel with the perfusion system so that the perfusion pressure could be maintained constant at $100 \mathrm{mmHg}$. The blood flow rate to the atrial preparation was 4 to $7 \mathrm{ml} / \mathrm{min}$. The ventricular margin of the isolated atrium was fixed to a stainless steel bar and placed in a cup-shaped glass container, which was maintained at a constant temperature of $37^{\circ} \mathrm{C}$ by means of a heating bath circulator (Haake, FE2). The upper part of the isolated atrium was connected to a force-displacement transducer (Nihon Kohden, AP620G) by a silk thread to record the isometric developed tension. The atrial muscle was usually stretched to a resting tension of $2 \mathrm{~g}$. A bipolar electrode was sewn on the epicardial surface of the atrium to record the atrial electrogram, and the spontaneous atrial rate of the atrium was derived from the electrogram with a cardiotachometer (Nihon Kohden, AP600G). The perfused blood was collected in a blood-reservoir and returned to the support dog via the left jugular vein. Sodium heparin (200 USP units $/ \mathrm{kg}$ ) was given intravenously at $1-\mathrm{hr}$ intervals.

The effects of AQ-A 39 on the ventricular contractility were studied in 3 isolated and electrically paced left ventricular preparations. The left ventricular muscle with the anterior descending branch of the left coronary artery was excised and the anterior descending branch was cannulated. The isolated preparation was perfused with heparinized arterial blood led from the donor dog, using the same circulating circuit which was used in the atrial preparation. Bipolar electrodes were sewn on the ventricular free wall. The ventricular muscle was driven electrically with rectangular pulses through the electrodes using an electrical stimulator (Nihon Kohden, SEN 7103). The stimulus pulse was $2 \mathrm{msec}$ duration, $6 \mathrm{~V}$ and $2 \mathrm{~Hz}$. The left ventricular developed tension was measured isometrically by a force-displacement transducer through a fine thread connected to the ventricular surface,

The heart rate derived from the electrocardiograph (lead II) and the femoral arterial blood pressure of the donor dog, plus the isometric developed tension and spontaneous atrial rate of the isolated preparation, were recorded 
on a thermal-writing rectigraph (Nihon Kohden, WT685G). Details of the isolated, blood-perfused dog right atrial and left ventricular preparations have been described in previous papers. ${ }^{11-13}$

The drugs used in the experiments were AQ-A 39 hydrochloride (Dr. Karl Thomae GmbH, Biberach), atropine sulfate (Wako Pure Chemical Ind., Osaka), d,l-propranolol hydrochloride (Sigma Chemical Co., St. Louis, MO), acetylcholine chloride (Daiichi, Tokyo), norepinephrine hydrochloride (Sankyo, Tokyo), isoproterenol hydrochloride (Nikken Chemicals, Tokyo), alinidine hydrobromide (Boehringer Ingelheim, Hyogo) and verapamil hydrochloride (Knoll A.G., Ludwigshafen). Drugs were dissolved in physiological saline before starting the experiments. Drugs were injected into the sinus node artery of the isolated atrium and into the anterior descending branch through a rubber tube with a microsyringe (Terumo Co., Tokyo) or administered into the external jugular vein of the donor dog. The amount of drug solution injected into the sinus node artery was 0.01 to $0.03 \mathrm{ml}$ over a period of $4 \mathrm{sec}$.

Summarized data are expressed as percentage of changes in maximum responses to each substance. Analysis of variance was used for the multiple comparisons of data and Student's t-test for paired data was used for comparison before and after antagonist treatment. $\mathbf{P}$ values of less than 0.05 were considered statistically significant.

\section{Results}

Cardiovascular effects of intravenous administration of AQ-A 39 in intact dogs and on isolated, blood-perfused atrial preparations:

Figure 1 shows the effects of intravenous injection of AQ-A $39(300 \mu \mathrm{g} / \mathrm{kg})$ into the external jugular vein of a donor dog. AQ-A 39 decreased the heart rate of the donor dog consistently but did not change atrial contractility. About $1.5 \mathrm{~min}$ after an intravenous injection of AQ-A 39, the atrial rate of the isolated atrium decreased because the drug injected into the donor dog perfused the isolated atrium. The devcloped tension of the isolated atrium also increased slightly and transiently. At doses of $10-100 \mu \mathrm{g} / \mathrm{kg}$, i.v., AQ-A 39 dose-dependently decreased both the heart rate of the donor dog and the atrial rate of the isolated atrium (Fig. 2). The durations of the decreases in heart rate and atrial rate at $300 \mu \mathrm{g} / \mathrm{kg}$ i.v. were approximately 30 and 20 min, respectively. AQ-A 39 at $1,000 \mu \mathrm{g} / \mathrm{kg}$ i.v. decreased the heart rate and atrial rate by approximately $20 \%$ from the control levels. On the other hand, these i.v. doses of AQ-A 39 revealed a biphasic response, consisting of a transient decrease followed by an increase in mean arterial blood pressure 

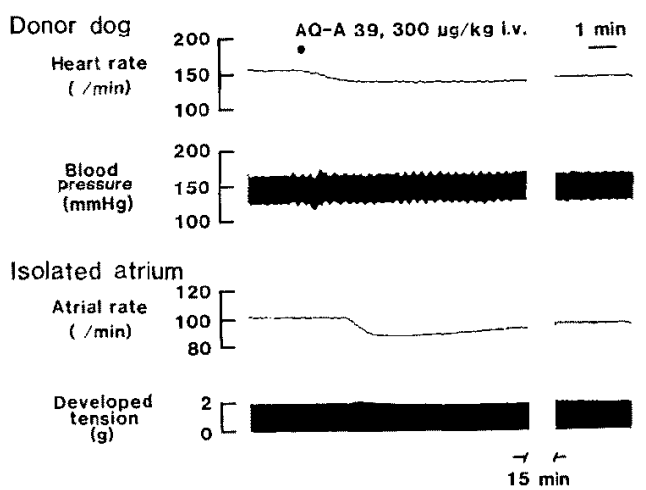

Fig. 1. Effects of intravenous administration of AO-A $39(300 \mu \mathrm{g} / \mathrm{kg})$ on the heart rate and systemic blood pressure of a donor dog (upper panel) and on the atrial rate and developed tension in an isolated dog atrium (lower panel) perfused with arterial blood of the donor dog.

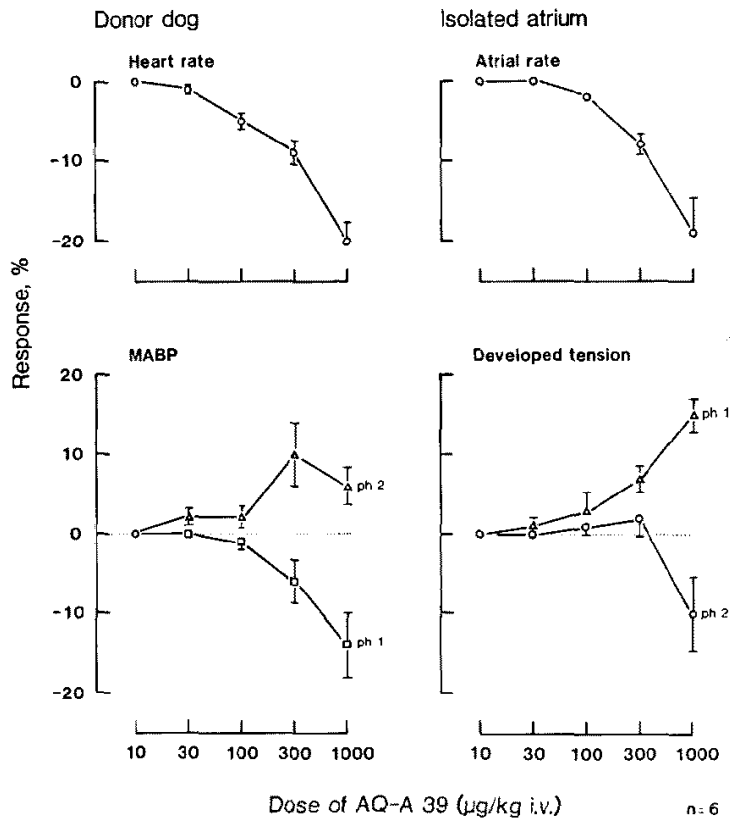

Fig. 2. AQ-A 39 dose-response curves for percentage changes in the heart rate and mean arterial blood pressure (MABP) of the donor dog (left panel) and in the atrial rate and developed tension of the isolated, bloodperfused atrium (right panel) in 6 experiments. AQ-A 39 was injected intravenously into the donor dog at doses of $10-1,000 \mu \mathrm{g} / \mathrm{kg}$. The control levels of heart rate and MABP of the donor dog werc $134 \pm 11$ (mean \pm SEM) beats/ min and $85 \pm 12.5 \mathrm{mmHg}$, respectively. The control levels of atrial rate and developed tension of the isolated atrium were $92 \pm 9$ beats $/ \mathrm{min}$ and $1.8 \pm 0.2 \mathrm{~g}$, respectively. Vertical bars show SEM. See text for explanation of ph 1 and ph 2. 
(MABP). In Fig. 2 (left panel), the transient decrease and increase in MABP were indicated as "ph 1" and "ph 2", respectively. After these temporary, biphasic changes in MABP, MABP was returned to the predrug level and maintained constant during the AQ-A 39-induced bradycardia.

In the isolated atrial preparation, AQ-A 39 at doses of $10-1,000 \mu \mathrm{g} / \mathrm{kg}$ i.v. caused a transient increase in developed tension, indicated as " $\mathrm{ph} 1$ " in Fig. 2 (right panel). After this temporary positive inotropic effect, though, there were no significant changes in contractility up to $300 \mu \mathrm{g} / \mathrm{kg}$ i.v. However, AQ-A 39 at $1,000 \mu \mathrm{g} / \mathrm{kg}$ i.v. elicited a slight negative inotropic effect $(\mathrm{p}<0.05)$ as indicated by "ph 2 " in Fig. 2 (right panel).

Effects of $A Q-A 39$ on pacemaker activity and atrial contractility in the isolated, blood-perfused dog atrium:

The effects of a direct injection of a dose of 10 or $100 \mu \mathrm{g}$ of AQ-A 39 into the sinus node artery of an isolated atrium are shown in Fig. 3. Ten micrograms of AQ-A 39 elicited a negative chronotropic and a small positive inotropic effect, while $100 \mu \mathrm{g}$ of AQ-A 39 produced a marked negative chronotropic response and a slight negative inotropic response which followed a transient positive inotropic reaction. Data comparing the effects of AQ-A 39 , alinidine and verapamil on pacemaker activity and contractility in isolated atria are presented in Fig. 4. The verapamil effects on the isolated and blood-perfused preparation are cited from Chiba. ${ }^{14}$ ) When doses of 1-300 $\mu \mathrm{g}$ of AQ-A 39 were injected directly into the sinus node artery of the isolated, blood-perfused dog atrium, a negative chronotropic effect was produced in a dose-dependent manner (Fig. 4). The drug also induced a small negative inotropic effect following a transient positive inotropic effect. Another specific bradycardic agent, alinidine, at doses of $1-300 \mu \mathrm{g}$, elicited dose-dependent negative chronotropic and inotropic responses (Fig. 4, open circles). Verapamil also produced marked negative inotropic and chronotropic effects in a dosc-related fashion. The order of the depth of bradycardia was ve-

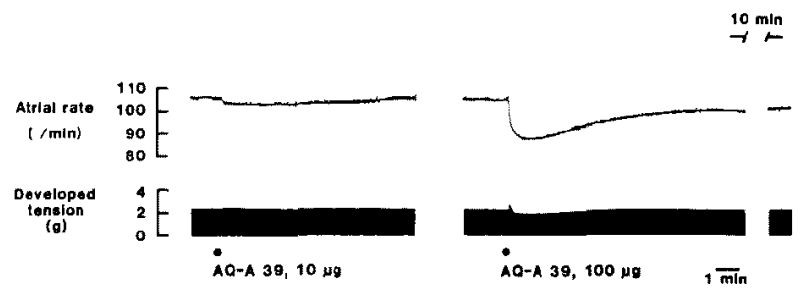

Fig. 3. The effects of injections of AQ-A 39 (10 or $100 \mu \mathrm{g}$ ) into the sinus node artery of an isolated and perfused dog atrium on atrial rate and developed tension. 


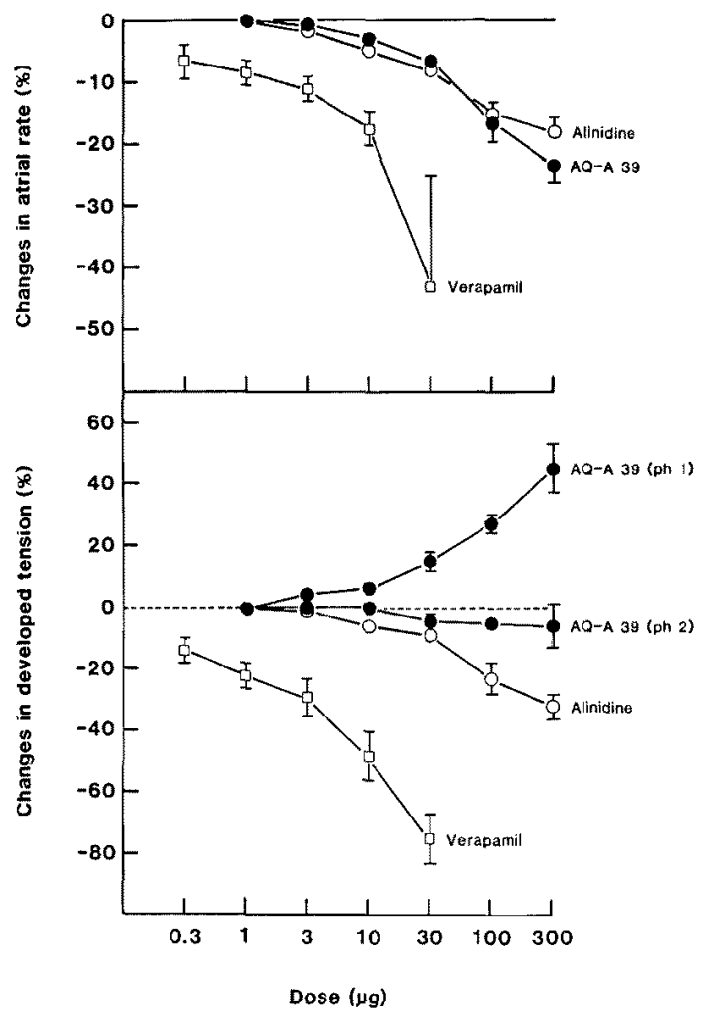

Fig. 4. Dose-response curves of percent changes in atrial rate and developed tension after direct injection of $\mathrm{AQ}-\mathrm{A} 39$, alinidine and verapamil into the sinus node artery of the isolated and perfused dog atrium in 5-7 experiments. The control levels of atrial rate and developed tension were $99 \pm 9$ (mean \pm SEM) beats $/ \mathrm{min}$ and $1.9 \pm 0.2 \mathrm{~g}$, respectively. Vertical bars show SEM.

rapamil $\gg A Q-A \quad 39 \geq$ alinidine. Unlike $A Q-A 39$ and alinidine, verapamil caused sinus arrest in 4 out of 7 preparations at $30 \mu \mathrm{g}$ and in all 3 preparations at $100 \mu \mathrm{g}$. The threshold doses for the negative inotropic effects of verapamil, alinidine and AQ-A 39 were approximately $0.3,3$ and $30 \mu \mathrm{g}$, respectively. The negative inotropic effect of $A Q-A 39$ was quite small in comparison with those of alinidine and verapamil. Furthermore, unlike alinidine and verapamil, the percentage of attenuation of decreases in chronotropic effects of AQ-A 39 was always greater than for inotropic effects.

In 3 electrically paced isolated and blood-perfused dog left ventricular preparations, AQ-A $39(1-300 \mu \mathrm{g})$ caused only a slight, transient positive inotropic response (data not shown). 
856 OGIWARA, FURUKAWA, AKAHANE, HANIUDA, AND CHIBA Jpo Heart J.

Pharmacological analyses of chronotropic and inotropic responses to $A Q-A 39$ in the isolated atrium:

Ten micrograms of atropine, a potent muscarinic antagonist, completely inhibited the negative chronotropic and inotropic effects of $0.3 \mu \mathrm{g}$ of acetylcholine. However, the negative chronotropic and inotropic effects of $300 \mu \mathrm{g}$ of AQ-A 39 were not significantly affected in 6 isolated atria (Fig. 5). Atropine $(10 \mu \mathrm{g})$ treatment did not alter the basal atrial rate or the developed tension.

Treatment with $10 \mu \mathrm{g}$ of propranolol, a beta-adrenoceptor antagonist, consistently inhibited norepinephrine (NE) $(0.03 \mu \mathrm{g})$-induced positive chronotropic and inotropic effects in isolated atria. This dose of propranolol slightly but significantly $(\mathrm{p}<0.05)$ potentiated the negative chronotropic response to AQ-A 39 (Fig. 6). The small negative inotropic response to AQ-A 39 was not enhanced significantly, and the transicnt positive inotropic response to AQ-A 39 was not affected.
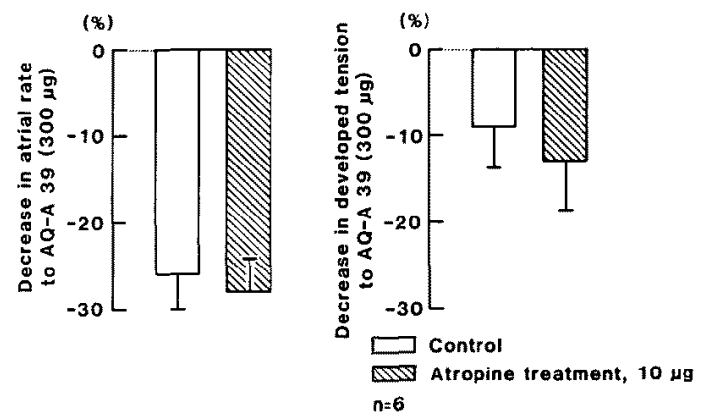

Fig. 5. Effects of atropine treatment $(10 / \mathrm{g})$ on decreases in atrial rate and developed tension in response to AQ-A $39(300 \mu \mathrm{g})$. Data are taken from 6 isolated and blood-perfused dog atria.
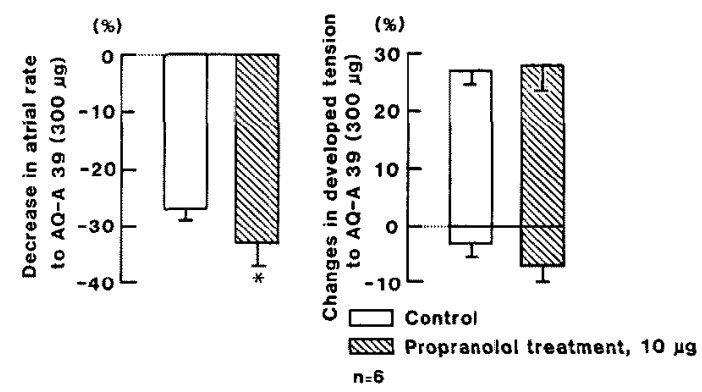

Fig. 6. Effects of propranolol $(10 \mu \mathrm{g})$ on chronotropic and inotropic responses to AQ-A $39(300 \mu \mathrm{g})$. Data reflect results from 6 isolated and bloodperfused dog atria. $* \mathrm{p}<0.05$ compared to the respective control. 
Influences of AQ-A 39 on positive chronotropic and inotropic responses to catecholamines:

When NE (0.03 and $0.1 \mu \mathrm{g})$ or isoproterenol (ISP; 0.01 and $0.03 \mu \mathrm{g}$ ) were injected into the sinus node artery of the isolated atrial preparation, positive chronotropic and inotropic effects were dose-dependently observed. When the sinus rate and atrial contractile force were decreased by $25 \pm 6.0$ and $14 \pm 6.0 \%$, respectively, by $300 \mu \mathrm{g}$ of $\mathrm{AQ}-\mathrm{A} 39$, the positive chronotropic effects of ISP and NE were also depressed slightly (Fig. 7). The effects of a small dose of $\mathrm{NE}(0.3 \mu \mathrm{g})$ were significantly attenuated by $300 \mu \mathrm{g}$ of AQ-A 39. On the other hand, the positive inotropic effects of ISP and NE were not affected.

The effects of verapamil ( 3 or $10 \mu \mathrm{g}$ ) on the positive cardiac responses to $\mathrm{NE}(0.03$ and $0.1 \mu \mathrm{g})$ were also tested in 4 experiments. When verapamil reduced both the sinus rate (about 15\%) and the atrial contractile force (about 50\%), it did not depress the positive chronotropic effect of NE. However, the positive inotropic effect of NE, expressed in $g$ but not in percentage changes, was depressed significantly $(\mathrm{p}<0.05)$.

\section{Discussion}

It has been reported that AQ-A 39 selectively decreased heart rate at
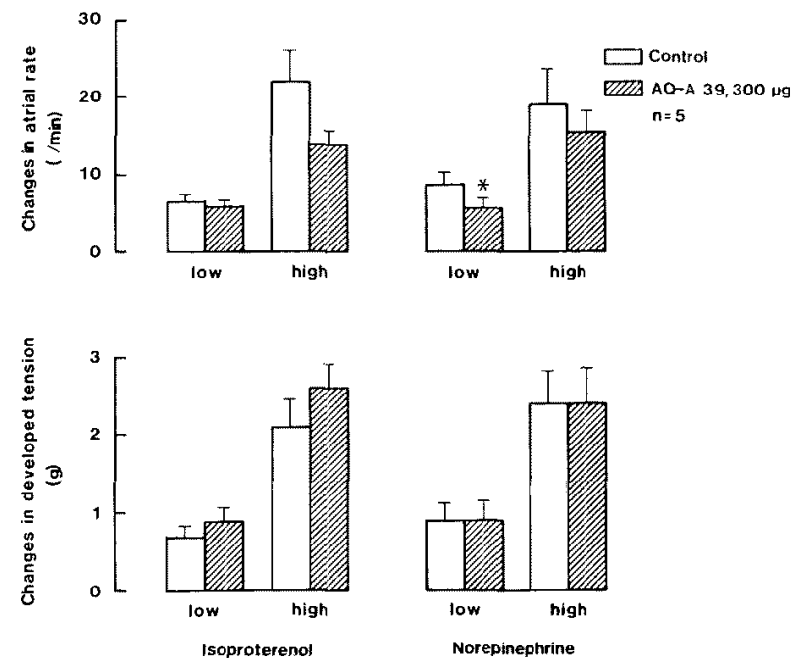

Fig. 7. Influences of AQ-A $39(300 \mu \mathrm{g})$ on positive chronotropic and inotropic responses to either isoproterenol [0.01 (low) and 0.03 (high) $\mu \mathrm{g}$ ], or norepinephrine $[0.03$ (low) and 0.1 (high) $\mu \mathrm{g}]$ in the isolated and bloodperfused dog atrium. $* \mathrm{p}<0.05$ compared to the respective control. 
doses which did not produce any other prominent cardiovascular effects. ${ }^{1)-3), 99}$ Kobinger and Lillie ${ }^{2}$ reported that $5 \mathrm{mg} / \mathrm{kg}$ i.v. of AQ-A 39 markedly increased the heart period and the duration of diastole, but the decrease in blood pressure was small in chloralose anesthetized cats. In conscious dogs, thcy also observed that an intravenous injection of AQ-A 39 markedly decreased the heart rate but had no effect on the blood pressure. In the present study, intravenous administration of AQ-A 39 to the anesthetized donor dog caused dose-dependent decreases in both the heart rate of the donor dog and the atrial rate of the isolated atrium. On the other hand, an intravenous injection of AQ-A 39 had no significant tonic effect on blood pressure, after the conclusion of transient biphasic effects. Furthermore, AQ-A 39 (up to $300 \mu \mathrm{g} / \mathrm{kg}$ i.v.) caused bradycardia with no significant inotropic changes, after the transient increase in atrial developed tension in the isolated atrium. Thus, AQ-A 39 selectively decreased the heart rate, in comparison with the changes in blood pressure and cardiac contractility in anesthetized dogs. The decreases in heart rate of the donor $\operatorname{dog}$ and in atrial rate of the isolated atrium were approximately of the same degree, referenced as percentage decreases from control values. Therefore, we confirmed that a site of the bradycardic actions of AQ-A 39 may be the sinus node in the dog heart.

Verdouw et al ${ }^{15}$ ) reported that AQ-A 39 (total dose $5.25 \mathrm{mg} / \mathrm{kg}$ i.v.) in the anesthetized pig markedly decreased the heart rate and slightly decreased the myocardial contractility; systemic vascular resistance was not affected. Siegl et $\mathrm{a}^{\left.\mathbf{l}^{6}\right)}$ observed that AQ-A 39 decreased the vascular resistance and increased the stroke volume in both non-paced and paced dog preparations, but a significant change in left ventricular $\mathrm{dP} / \mathrm{dt}_{\max }$ was not observed. From those results, they suggested that the augmented stroke volume in the paced preparation was probably due to a reduction in afterload by AQ-A 39. In the present study, the blood pressure of the donor dog was not affected significantly by AQ-A 39 , except for transient effects immediately after the drug injection. Furthermore, the atrial contractility of the preparation did not consistently change with AQ-A 39. Although we did not measure the longterm effects on total vascular resistance, if the vascular resistance was markedly decreased by AQ-A 39, the blood pressure would probably decrcase. Therefore, the decrease in total vascular resistance with AQ-A 39 appears to be relatively small in the anesthetized dog.

Direct injection of AQ-A 39 into the sinus node artery of the isolated atrial preparation produced a negative chronotropic effect; no significant negative inotropic effect following a transient positive one. On the other hand, alinidine caused marked negative chronotropic and inotropic effects. Kobinger and Lillie $^{2}$ observed the predominant bradycardic effect of AQ-A 39 
and alinidine in isolated guinea pig atria and concluded that the responses to AQ-A 39 and alinidine were similar. Kawada et $\mathrm{al}^{3 \text {,16) }}$ reported that both AQ-A 39 and alinidine produced negative chronotropic effects in isolated, blood-perfused heart preparations from dogs and that both drugs at higher doses caused negative inotropic effects in the isolated, blood-perfused papillary muscle. In the present study, AQ-A 39 always elicited a negative chronotropic effect, but a decrease in contractility was not consistent. On the other hand, alinidine showed both negative chronotropic and inotropic effects. Thus, using isolated and blood-perfused canine atrial preparations, we demonstrated that AQ-A 39 differed from alinidine with respect to its actions on cardiac contractility.

It was reported that the AQ-A 39-induced bradycardia partly reflects actions on slow calcium channels in electrophysiological experiments. ${ }^{7,8}$ This is consistent with its structural similarities to verapamil. However, the chronotropic and inotropic responses to verapamil were different from those of AQ-A 39 in isolated canine atria. Both drugs caused similar negative chronotropic effects, but verapamil produced a marked depression of cardiac contractility and sinus arrest. Therefore, the calcium antagonistic effects of AQ-A 39 may be disregarded in the dog heart. ${ }^{3}$ )

This study confirmed that the negative cardiac effects of AQ-A 39 in the dog heart are not mediated by modification of muscarinic cholinergic receptors. As reported in the guinea-pig," the negative chronotropic and inotropic effects of AQ-A 39 were not significantly affected by an adequate dose of atropine. Sys et $\mathrm{al}^{5}{ }^{5}$ reported that low concentrations of AQ-A 39 could produce a positive inotropic effect in the cat papillary muscle. Urthaler and Walker ${ }^{4}$ also demonstrated a positive inotropic effect of AQ-A 39 in isolated dog ventricles. In their experiments, the positive inotropic effect of AQ-A 39 was significantly reduced by propranolol treatment. Thus, they concluded that this positive inotropic effect was due, in part to neuronal norepinephrine release.

The negative chronotropic effects of $\Lambda Q-\Lambda 39$ in the present experiments were significantly enhanced by propranolol treatment, suggesting that the AQ-A 39-induced bradycardia is antagonized, in part, by beta-adrenoceptor activity. The slight negative inotropic effect of AQ-A 39 also tended to be potentiated by propranolol, but not significantly. On the other hand, the transient positive inotropic effect was not affected by propranolol, suggesting that it is independent of beta-adrenoceptors. From these results, it is considered that AQ-A 39-induced cardiac responses are modified, at least in part, by actions of beta-receptors in the dog heart.

It has been reported that AQ-A 39 and alinidine depressed isoproterenol- 
induced tachycardia, but that propranolol suppressed both positive chronotropic and inotropic responses to isoproterenol in guinea pig atria. ${ }^{91}$ Wenger and Sieglin ${ }^{17)}$ also reported that AQ-A 39 had a greater selectivity for inhibiting the chronotropic responses to beta-adrenergic stimulation than verapamil or timolol in the anesthetized dog. In the present study, the positive chronotropic responses to low doses of norepinephrine were significantly attenuated by AQ-A 39 treatment. However, the positive inotropic responses to these catecholamines were not depressed at all. We recently observed that alinidine attenuated increases in sinus rate in response to either catecholamines or dibutyryl cyclic AMP, without affecting increases in contractile force in response to those agents in isolated and perfused dog atria. ${ }^{10)}$ The selective attenuation of the NE-induced positive chronotropic effect of AQ-A 39 was similar to the attenuation induced by alinidine. By contrast, verapamil significantly depressed the NE-induced positive inotropic response, but not the chronotropic response. Propranolol, though, inhibited both positive chronotropic and inotropic responses to NE. These results suggest that the selective attenuation of the NE-induced positive chronotropic effect by AQ-A 39 or alinidine is related to a specific bradycardic property, which is independent of beta-adrenoceptor blockade and calcium channel antagonism in the isolated dog atrium.

\section{RefERENCES}

1. Dammgen J, Kadatz R, Diederen W: Cardiovascular actions of 5,6-dimethoxy-2- $\{3-[(\alpha-$ (3,4-dimethoxy)phenylethyl)methylamino]propyl) phthalimidine (AQ-A 39), a specific bradycardic agent. Arzneim-Forsch $31: 666,1981$

2. Kobinger W, Lillie G: AQ-A 39 (5,6-dimethoxy-2[3[[ $\alpha$-(3,4-dimethoxy)-phenylethyl]methylamino]propyl]phthalimidine), a specific bradycardic agent with direct action on the heart. Eur J Pharmacol 72: 153, 1981

3. Kawada M, Satoh K, Taira N: Analysis of the cardiac action of the bradycardic agent, AQ-A 39, by use of isolated, blood-perfused dog-heart preparations. J Pharmacol Exp Ther 228: 484,1984

4. Urthaler $F$, Walker AA: Indirect stimulatory action of the calcium channel blocker AQ-A 39. J Pharmacol Exp Ther 230: 336, 1984

5. Sys SU, DeClerck NM, Brutsaert DL: Effects of AQ-A 39 on contractile performance of isolated mammalian ventricular myocardium. Eur J Pharmacol 103: 33, 1984

6. Siegl PKS, Wenger HC, Sweet CS: Comparison of cardiovascular responses to the bradycardic drugs, alinidine, AQ-A 39, and mixidine, in the anesthetized dog. J Cardiovasc Pharmacol 6: 565, 1984

7. Trautwein W, Pelzer D, McDonald TF, Osterrieder W: AQ-A 39, a new bradycardic agent which blocks myocardial calcium (Ca) channels in a frequency- and voltage-dependent manner. Naunyn-Schmiedeb Arch Pharmacol 317: 228, 1981

8. Osterrieder W, Pelzer D, Yang Q-F, Trautwein W: The electrophysiological basis of the bradycardic action of AQ-A 39 on the sinoatrial node. Naunyn-Schmiedeb Arch Pharmacol 317: 233,1981 
9. Lillie $\mathrm{C}$, Kobinger W: Actions of alinidine and AQ-A 39 on rate and contractility of guinea pig atria during $\beta$-adrenoceptor stimulation. J Cardiovasc Pharmacol 5: 1048, 1983

10. Ogiwara Y, Furukawa Y, Saegusa K, Takeda M, Chiba S: Bradycardic effect of alinidine on in situ and on isolated, blood-perfused heart preparations of the dog. Arch Int Pharmacodyn Ther 291: 41, 1988

11. Chiba S, Kimura T, Hashimoto K: Muscarinic suppression of the nicotinic action of acetylcholine on the isolated, blood-perfused atrium of the dog. Naunyn-Schmiedeb Arch Pharmacol 289: 315, 1975

12. Chiba S, Yabuuchi Y, Hashimoto K: Comparison of the effects of norepinephrine and acetylcholine between intraarterial and extravascular administration to the isolated, bloodperfused canine atrium. Jpn J Pharmacol 25: 433, 1975

13. Chiba S: Effects of pentobarbital, verapamil and manganese on the frequency-force relationship of the isolated atrium and ventricle of the dog heart. Eur J Pharmacol 40: 225, 1976

14. Chiba S: Effects of verapamil on the blood-perfused, isolated atrium preparation of the dog heart. Jpn Heart J 16: 709, 1975

15. Verdouw PD, Bom HPA, Bijileveld RE: Cardiovascular responses to increasing plasma concentration of AQ-A 39, a new compound with negative chronotropic effects. ArzneimForsch 33: 702, 1983

16. Kawada M, Satoh K, Taira N: Selectivity of alinidine, a bradycardic agent, for SA nodal automaticity versus other cardiac activities in isolated, blood-perfused dog-heart preparations. Arch Int Pharmacodyn Ther 272: 88, 1984

17. Wenger HG, Siegl PKS: Influence of AQ-A 39, a bradycardic agent, on cardiovascular responses to isoproterenol in the anesthetized dog. J Cardiovasc Pharmacol 9: 246, 1987 